Testing the race model inequality in redundant stimuli with variable onset asynchrony

Gondan, Matthias

Published in:

Journal of Experimental Psychology: Human Perception and Performance

DOI:

$10.1037 / \mathrm{a} 0013620$

Publication date:

2009

Document version

Publisher's PDF, also known as Version of record

Citation for published version (APA):

Gondan, M. (2009). Testing the race model inequality in redundant stimuli with variable onset asynchrony. Journal of Experimental Psychology: Human Perception and Performance, 35(2), 575-579.

https://doi.org/10.1037/a0013620 


\title{
OBSERVATIONS
}

\section{Testing the Race Model Inequality in Redundant Stimuli With Variable Onset Asynchrony}

\author{
Matthias Gondan \\ University of Regensburg
}

\begin{abstract}
In speeded response tasks with redundant signals, parallel processing of the signals is tested by the race model inequality. This inequality states that given a race of two signals, the cumulative distribution of response times for redundant stimuli never exceeds the sum of the cumulative distributions of response times for the single-modality stimuli. It has been derived for synchronous stimuli and for stimuli with stimulus onset asynchrony (SOA). In most experiments with asynchronous stimuli, discrete SOA values are chosen and the race model inequality is separately tested for each SOA. Due to the high number of statistical tests, Type I and II errors are increased. Here a straightforward method is demonstrated to collapse these multiple tests into one test by summing the inequalities for the different SOAs. The power of the procedure is substantially increased by assigning specific weights to SOAs at which the violation of the race model prediction is expected to be large. In addition, the method enables data analysis for experiments in which stimuli are presented with SOA from a continuous distribution rather than in discrete steps.
\end{abstract}

Keywords: multisensory processing, race model inequality, race model, coactivation model

Supplementary materials: http://dx.doi.org/10.1037/a0013620.supp

In everyday perception the sensory systems simultaneously receive information. Although each sensory system projects to its specialized unisensory brain region, we generally have a unified and holistic representation of the environment. Therefore, the information provided by the sensory systems has to be integrated in some way. This process is called multisensory integration. The integration process can be investigated by providing redundant information via different sensory channels and by testing whether a redundancy gain is observed. In the basic experimental setup (redundant signals task; Miller, 1982), the same response is required for two stimuli: auditory (A) and visual (V). The stimuli are presented either as single targets or in combination (redundant targets; AV). It is often observed that responses to AV are faster than responses to $\mathrm{A}$ and $\mathrm{V}$ alone (redundant signals effect).

Different models explain the redundant signals effect. Consider, for example, serial exhaustive processing of AV: Upon presentation of the stimulus, A is processed, and when A is finished, $\mathrm{V}$ is processed. In this model, the processing time for AV is the sum of the processing times for $\mathrm{A}$ and $\mathrm{V}$. If only one target is presented (e.g., A, together with a visual nontarget, $\mathrm{V}^{0}$ ), the total processing time amounts to $T_{\mathrm{A}}+T_{\mathrm{V} 0}$. Provided that detection of a nontarget

Program code for the nonparametric test is available for download as online supplementary material.

Correspondence concerning this article should be addressed to Matthias Gondan, Department of Experimental Psychology, University of Regensburg, D-93050 Regensburg, Germany. E-mail: matthias.gondan@psychologie .uni-regensburg.de is slower than detection of a target, serial exhaustive processing of the stimuli predicts that responses to redundant targets are faster than responses to single targets (Townsend \& Nozawa, 1997; a similar argument holds if processing is serial but terminates as soon as the first target has been identified).

When participants are asked to simply press a button when they hear a sound or see an LED flash, parallel models seem to be more plausible accounts of information processing. An important model of parallel processing is the race model. It assumes separate processing of $\mathrm{A}$ and $\mathrm{V}$. Detection occurs if one of the two channels has finished processing (Miller, 1982):

$$
T_{\mathrm{AV}}=\min \left(T_{\mathrm{A}}, T_{\mathrm{V}}\right) .
$$

If $\mathrm{A}$ and $\mathrm{V}$ are presented with stimulus onset asynchrony (SOA)e.g., $\mathrm{V}$ following A by $\tau \mathrm{ms}, \mathrm{AV}(\tau)$ — the delay of the second stimulus needs to be taken into account (Miller, 1986):

$$
T_{\mathrm{AV}(\tau)}=\min \left(T_{\mathrm{A}}, T_{\mathrm{V}}+\tau\right) .
$$

Without loss of generality, only $\operatorname{AV}(\tau)$ is treated in the following. If $\mathrm{A}$ follows $\mathrm{V}(\tau<0)$, the indices in Equation 2 are simply exchanged. A response to $\operatorname{AV}(\tau)$ is faster than a given $t$ if one of the two processes has finished within $t$ :

$$
\begin{aligned}
\left\{T_{\mathrm{AV}(\tau)}<t\right\}=\left\{\min \left(T_{\mathrm{A}}, T_{\mathrm{V}}+\tau\right)<t\right\} & \\
= & \left\{T_{\mathrm{A}}<t\right\} \cup\left\{T_{\mathrm{V}}+\tau<t\right\} .
\end{aligned}
$$

Under the assumption that, regardless of $\tau$, the processing time distributions for $T_{\mathrm{A}}$ and $T_{\mathrm{V}}$ can be estimated through the unimodal response time distributions (context independence; Luce, 1986, p. 
130), limits for the probability for a fast response to $\operatorname{AV}(\tau)$ can be derived from Equation 3:

$$
\begin{gathered}
P\left\{T_{\mathrm{AV}(\tau)}<t\right\} \geq P\left\{T_{\mathrm{A}}<t\right\}, \\
P\left\{T_{\mathrm{AV}(\tau)}<t\right\} \geq P\left\{T_{\mathrm{V}}+\tau<t\right\}, \\
P\left\{T_{\mathrm{AV}(\tau)}<t\right\} \leq P\left\{T_{\mathrm{A}}<t\right\}+P\left\{T_{\mathrm{V}}+\tau<t\right\} .
\end{gathered}
$$

The upper bound in Inequality 4B is restated through the empirical cumulative distribution function

$$
F_{\mathrm{AV}(\tau)}(t) \leq F_{\mathrm{A}}(t)+F_{\mathrm{V}}(t-\tau) .
$$

In most experiments with redundant targets, the mean response times for redundant targets are below the mean response times for single targets, and only the upper bound is tested (race model inequality; e.g., Miller, 1986). If Inequality 5 is violated$F_{\mathrm{AV}(\tau)}(t)>F_{\mathrm{A}}(t)+F_{\mathrm{V}}(t-\tau)$-it is concluded that coactivation has occurred.

Specific coactivation models have been developed by Miller (1982, appendix), Miller (1986, Equation 3), Miller and Ulrich (2003, p. 116f), and Schwarz (1989, 1994). Although it is not explicitly mentioned in all of these studies, systematic variation of the SOA of A and V is essential for testing the majority of these models. For example, Miller (1986, pp. 332-334) derived an inequality for coactivation models with exponentially distributed detection times:

$$
F_{\mathrm{AV}(\tau)}(t) \leq F_{\mathrm{A}}(t)+F_{\mathrm{AV}}(t-\tau) .
$$

Rejection of Inequality 6 rules out exponential stimulus detection, favoring models in which the sensory channels accumulate evidence over time. Testing Inequality 6 requires asynchronous stimuli. In the diffusion superposition model (DSM; Schwarz, 1994), accumulation of sensory evidence is described by a diffusion process with drift; detection occurs when a criterion is reached for the first time. In redundant stimuli the criterion is reached earlier because both processes contribute to the buildup of activation. Schwarz (1994, p. 510) mentioned that the test of the DSM requires systematic variation of the SOA, including audition-first and vision-first conditions. Finally, Ulrich and Miller (1997) have shown that the slope of the function $\tau \rightarrow E\left[T_{\mathrm{AV}(\tau)}\right]$ can be used to distinguish between different classes of coactivation models. The examples underline the importance of SOA variation, especially if coactivation models are considered. In short, comparing AV to A and $\mathrm{V}$ enables testing whether or not the race model holds, whereas SOA variation yields insight into successive stages of the coactivation process.

In typical experiments, discrete SOAs are chosen (e.g., A167V, $\mathrm{A} 133 \mathrm{~V}, \ldots, \mathrm{V} 167 \mathrm{~A})$, and the race model inequality is tested for each SOA. The high number of significance tests results in an increased Type I error-or, if Bonferroni correction is applied $\left(\alpha^{\prime}=\alpha / n\right)$, the power of the test is greatly diminished. In the present note it is shown how Inequality 5 is tested across different SOAs, resulting in only one significance value. With Inequality 5 rearranged,

$$
F_{\mathrm{AV}(\tau)}(t)-F_{\mathrm{A}}(t)-F_{\mathrm{V}}(t-\tau) \leq 0 .
$$

Because Inequality 7 should hold for every SOA, it is possible to sum it over all values of $\tau$ :

$$
\Sigma_{\tau}\left[F_{\mathrm{AV}(\tau)}(t)-F_{\mathrm{A}}(t)-F_{\mathrm{V}}(t-\tau)\right] \leq 0 .
$$

In Inequality 8 , each SOA has equal weight, and a race model violation at one SOA might be canceled out by other SOAs in which $F_{\mathrm{AV}(\tau)}(t)$ is smaller than $F_{\mathrm{A}}(t)+F_{\mathrm{V}}(t-\tau)$. Equal weights are not required, though: If $a<b$ and $c<d$, then $\lambda_{1} a+\lambda_{2} c<$ $\lambda_{1} b+\lambda_{2} d$ for $\lambda_{1}, \lambda_{2}>0$. It is thus possible to assign different weights to different SOAs with a function $\lambda(\tau) \geq 0$ :

$$
\Sigma_{\tau} \lambda(\tau) \times\left[F_{\mathrm{AV}(\tau)}(t)-F_{\mathrm{A}}(t)-F_{\mathrm{V}}(t-\tau)\right] \leq 0 .
$$

Although, formally, every function $\lambda(\tau) \geq 0$ is allowed in Inequality 9 , the power of the test is increased if $\lambda(\tau)$ emphasizes SOAs at which coactivation effects are expected to be high, and small weights are assigned to SOAs in which coactivation effects are expected to be small: $\lambda\left(\tau_{1}\right)=0$ completely removes SOA $\tau_{1}$ from the analysis. Which weighting function should be used? Integration of the sensory signals has been shown to be optimal if stimuli are presented in close temporal proximity (Meredith, Nemitz, \& Stein, 1987). Coactivation effects might thus be expected to be highest around $\tau=0$, suggesting an umbrella-shaped $\lambda(\tau)$ around $\tau=0$ (see below). In contrast, Miller (1986, p. 337) noted that for auditory-visual stimuli, "the SOAs producing the largest violations of race models are approximately those that equalize mean RTs to single signals." Hence, a "shifted umbrella" around mean $\left(T_{\mathrm{V}}\right)-\operatorname{mean}\left(T_{\mathrm{A}}\right)$ might be appropriate to emphasize the SOA at which the distributions of $T_{\mathrm{A}}$ and $T_{\mathrm{V}}$ have a high chance to overlap (physiological synchrony; Hershenson, 1962). Further estimates for $\lambda(\tau)$ can be derived from coactivation models (e.g., the DSM; Schwarz, 1994; see supplementary material).

\section{Statistical Tests}

Several approaches have been suggested to test the race model prediction. The present method can be applied to each of these tests. In the simplest approach, data are collected from a number of participants and Inequality 5 is tested with the binary information of each participant (whether it holds at a given SOA and $t$ ). A binomial test seems appropriate here, because under the race model assumption, the maximum redundancy gain is $F_{\mathrm{AV}(\tau)}(t)=F_{\mathrm{A}}(t)+F_{\mathrm{V}}(t-\tau)$ (Maris \& Maris, 2003). Due to sampling error, $F_{\mathrm{AV}(\tau)}(t)>F_{\mathrm{A}}(t)+F_{\mathrm{V}}(t-\tau)$ in half of the participants. The race model is rejected if the observed proportion of participants showing violations of Inequality 5 is significantly higher than 50\%. An aggregate test for A70V, AV V70A might use a symmetric umbrella around $\tau=0$, testing if $1 \times F_{\mathrm{A} 70 \mathrm{~V}}(t)+2 \times F_{\mathrm{AV}}(t)+1 \times F_{\mathrm{V} 70 \mathrm{~A}}(t)$ is greater than $1 \times$ $\left[F_{\mathrm{A}}(t)+F_{\mathrm{V}}(t-70)\right]+2 \times\left[F_{\mathrm{A}}(t)+F_{\mathrm{V}}(t)\right]+1 \times\left[F_{\mathrm{A}}(t-\right.$ $\left.70)+F_{\mathrm{V}}(t)\right]$, for a given $t$. Although Inequality 5 should hold for every $t$, an appropriate $t$ should be chosen for the test (e.g., percentile 20 in condition AV; Kiesel, Miller, \& Ulrich, 2007). As for different values of $\tau$, it is possible to add up Inequality 5 for several $t$ s (e.g., 250, 300, $350 \mathrm{~ms}$ ) and thus avoid an increased Type I error when testing at more than one $t$ (Kiesel et al., 2007).

Most often, the race model is tested with the Vincentized (Jiang, Rouder, \& Speckman, 2004) data,

$$
F_{\mathrm{AV}(\tau)}^{-1}(p) \geq\left[F_{\mathrm{A}}+F_{\mathrm{V}}^{\tau}\right]^{-1}(p)
$$


that is, the response times at fixed percentiles are compared $\left(F^{-1}\right.$ denotes the inverse cumulative distribution, and $F_{\mathrm{V}}^{\tau}$ indicates that the distribution for the second stimulus is shifted by $\tau \mathrm{ms}$ ). Miller (1982) showed that Inequality 10 can be tested with a $t$ test for paired samples. If $F_{\mathrm{AV}(\tau)}^{-1}(p)$ is significantly below $\left[F_{\mathrm{A}}+\right.$ $\left.F_{\mathrm{V}}^{\tau}\right]^{-1}(p)$ for a given $p$, the race model is rejected. With Inequality 10 collapsed for different SOAs,

$$
\Sigma_{\tau} \lambda(\tau) \times\left\{F_{\mathrm{AV}(\tau)}^{-1}(p)-\left[F_{\mathrm{A}}+F_{\mathrm{V}}^{\tau}\right]^{-1}(p)\right\} \geq 0 .
$$

An aggregate test for the SOAs and weights above would test if $1 \times F_{\mathrm{A} 70 \mathrm{~V}}^{-1}(p)+2 \times F_{\mathrm{AV}}^{-1}(p)+1 \times F_{\mathrm{V} 70 \mathrm{~A}}^{-1}(p)$ were significantly below $1 \times\left[F_{\mathrm{A}}+F_{\mathrm{V}}^{70}\right]^{-1}(p)+2 \times\left[F_{\mathrm{A}}+F_{\mathrm{V}}\right]^{-1}(p)+1 \times$ $\left[F_{\mathrm{A}}^{70}+F_{\mathrm{V}}\right]^{-1}(p)$. Kiesel et al. (2007) recommended testing at percentiles 10-25. Again, the race model predicts that Inequality 11 holds for every $p$. Therefore, it is possible to perform a simultaneous test of more than one percentile by adding up Inequality 11 for different $p$ s.

Miller (1986) suggested a measure of the race model violation: the area $\Delta_{\tau}$ between $F_{\mathrm{AV}(\tau)}(t)$ and $F_{\mathrm{A}}(t)+F_{\mathrm{V}}(t-\tau)$, where $F_{\mathrm{AV}(\tau)}(t)>F_{\mathrm{A}}(t)+F_{\mathrm{V}}(t-\tau)$ :

$$
\Delta_{\tau}={ }_{0} \int^{\infty} \max \left[0, F_{\mathrm{AV}(\tau)}(t)-F_{\mathrm{A}}(t)-F_{\mathrm{V}}(t-\tau)\right] d t .
$$

According to the race model, this "violation area" should be 0 . To determine if an observed $\Delta_{\tau}>0$ reflects true coactivation or is due to sampling error, the distribution of $\Delta_{\tau}$ under the race model is generated by simulating the experiment, say, 1,000 times. In each simulation $i$, the response times for auditory and visual stimuli $\left(F_{\text {Asim }}, F_{\text {Vsim }}\right)$ are resampled with replacement from the observed auditory and visual response times, respectively (bootstrap). $F_{\text {AVsim }}$ is generated by sampling again from the auditory and visual response times and choosing the smaller of each response time pairing. To maximize the redundancy gain, fast responses to auditory stimuli are combined with slow responses to visual stimuli, that is, $F_{\mathrm{A}}^{-1}(p)$ with $F_{\mathrm{V}}^{-1}(1-p)$ (Miller, 1986, pp. 336-337). $\Delta_{\tau}(i)$ is then determined according to Equation 12. If the observed $\Delta_{\tau}$ is greater than $95 \%$ of the simulated areas $\Delta_{\tau}(i)$, a violation of the race model prediction is considered statistically significant at the $5 \%$ level.

This nonparametric test is readily generalized to multiple SOAs by collapsing the SOA-specific $\Delta_{\tau}$ into one overall $\Delta$ by a weighted sum (the maximum of the $\Delta_{\tau}$ might do as well):

$$
\begin{gathered}
\Delta=\Sigma_{\tau} \lambda(\tau) \times \Delta_{\tau}, \\
\Delta=\max _{\tau} \Delta_{\tau} .
\end{gathered}
$$

Under the race model, each $\Delta_{\tau}$ should be 0 ; therefore, the weighted sum or the maximum of the $\Delta_{\tau}$ should be 0 as well. Instead of the inequalities being added up as in Inequality 9 or 11 , the $\Delta_{\tau} \mathrm{s}$ are added up in Equation 13. As the violation area cannot be negative, the power of the test is improved: A given $\Delta_{\tau}>0$ for SOA $\tau$ is not canceled out by SOA $v$ in which $F_{\mathrm{AV}(v)}(t)<F_{\mathrm{A}}(t)+F_{\mathrm{V}}(t-v)$.

A few comments on the nonparametric test of Miller (1986) might be of interest. Although the race model predicts that $F_{\mathrm{AV}(\tau)}(t)-F_{\mathrm{A}}(t)-F_{\mathrm{V}}(t-\tau) \leq 0$ for all $t \mathrm{~s}$, the range of the integral in Equation 12 can be restricted to exclude fast guesses, such as $\left[F_{\mathrm{AV}}^{-1}(.05), \infty\right]$, left-censoring response times at the 5 th percentile of $F_{\mathrm{Av}}$. If the experiment is divided into sessions of 15-min duration, say, stratified resampling that uses sessions as strata further improves the test because different levels of overall speed are mirrored by the bootstrap samples (Davison \& Hinkley, 1997). Stratified bootstrapping requires enough trials per session (see Table 1).

If more than one participant took part in the experiment, metaanalysis techniques can be used to test the race model prediction in a group. Under the null hypothesis, $p$ values are uniformly distributed in $[0,1]$ and can be transformed to $\chi^{2}$ values: $\chi^{2}(d f=2)=$ $-2 \log P$ (Bulmer, 1979). The sum of $N$ independent $\chi^{2}$ can then be tested for significance $(d f=2 N)$. More sophisticated techniques involve weighting functions and truncation of extreme $p$ values (e.g., Saner, 1994).

The above examples focus on the classical race model test (Inequality 5). An aggregate test for Inequality 6 can be constructed for testing coactivation models with exponentially distributed detection times. Of course, a different weighting function would be used in this test. Finally, Miller (1986, pp. 337-338) suggested a procedure for comparing violation areas of two SOAs. This procedure is again readily generalized to weighted sums of violation areas (e.g., audition-first and vision-first stimuli). Both tests are described in the supplementary material.

\section{Application to Miller's (1986) Data and Simulation Results}

The new method was evaluated with existing data from Miller (1986). Two participants (B.D., K.Y.) made speeded responses to auditory-visual stimuli at 11 SOAs $(\mathrm{A} 167 \mathrm{~V}, \ldots, \mathrm{V} 167 \mathrm{~A}$, in 33-ms steps, 400 responses per SOA). Results of SOA-specific race model tests are shown in Miller (1986, Table 2). In B.D. the race model prediction was violated at a large range of SOAs; in K.Y. a violation of the race model was observed only in V67A and V100A.

The data were reanalyzed with the aggregate test (Equation 13) and different weighting functions: equal weights, umbrella around 0 , shifted umbrella around mean $\left(T_{\mathrm{V}}\right)-\operatorname{mean}\left(T_{\mathrm{A}}\right)$, weighting function derived from the DSM, and maximum violation area (Equation 14). For B.D. all aggregate tests were highly significant (see Table S1 in the supplementary material). For K.Y. the unweighted test was not significant $(p=.109)$, probably because significant violations in V67A and V100A were washed out by null effects in the other SOAs. In contrast, the shifted umbrella test, the test using DSM weights, and the test based on the maximum violation area (Equation 14) indicated significant violations of the race model $(p=.005, p=.019$, and $p=.040$, respectively). Assuming that coactivation occurred in both participants, the appropriate weight function seems to substantially increase the power of the test.

This is confirmed by 1,000 simulated experiments in which a coactivation model (Schwarz, 1994) was used to generate $N=30$, 90, and 300 auditory-visual detection times. To each detection time $D$, an exponentially distributed base time was added, reflecting peripheral processes and motor processes. The simulated experiment was divided into three sessions with different base times ( $\mu_{M}=10,20,30 \mathrm{~ms}$, respectively). Data were analyzed with Miller's (1986) nonparametric test, with sessions as strata for resampling. Table 1 shows the amount of significant $p$ values. It is evident that the power increases with sample size, and it increases further if an appropriate weighting function is used. To control the 
Table 1

Type I Error and Power of Miller's (1986) Test for Individual Stimulus Onset Asynchronies (SOAs) and Aggregate Tests With Different Weighting Functions

\begin{tabular}{|c|c|c|c|c|c|c|}
\hline \multirow[b]{2}{*}{ SOA } & \multicolumn{3}{|c|}{$\alpha$, race model $^{\mathrm{a}}$} & \multicolumn{3}{|c|}{ Power, coactivation model ${ }^{\mathrm{b}}$} \\
\hline & $N=3 \times 10$ & $N=3 \times 30$ & $N=3 \times 100$ & $N=3 \times 10$ & $N=3 \times 30$ & $N=3 \times 100$ \\
\hline A167V & .100 & .084 & .080 & .049 & .041 & .030 \\
\hline A133V & .090 & .096 & .093 & .048 & .044 & .043 \\
\hline A100V & .089 & .088 & .091 & .035 & .033 & .042 \\
\hline A67V & .089 & .085 & .090 & .047 & .042 & .073 \\
\hline A33V & .097 & .077 & .088 & .065 & .074 & .160 \\
\hline AV & .104 & .088 & .099 & .149 & .259 & .677 \\
\hline V33A & .098 & .077 & .085 & .225 & .503 & .956 \\
\hline V67A & .093 & .068 & .066 & .191 & .442 & .913 \\
\hline V100A & .113 & .086 & .072 & .144 & .199 & .526 \\
\hline V133A & $.123^{\mathrm{c}}$ & .096 & .108 & .090 & .093 & .165 \\
\hline V167A & .113 & .112 & .094 & .087 & .057 & .072 \\
\hline$\Sigma$ & .093 & .075 & .075 & .064 & .106 & .374 \\
\hline$\cap$ & .101 & .074 & .072 & .088 & .166 & .578 \\
\hline$\rightarrow \cap$ & .121 & .080 & .078 & .151 & .239 & .720 \\
\hline Max & .088 & .088 & .082 & .048 & .040 & .179 \\
\hline
\end{tabular}

Note. Stratified test for three sessions with exponentially distributed base times $\left(\mu_{M}=10,20,30 \mathrm{~ms}\right.$, respectively). $\Sigma=$ summed violation areas, equal weights; $\cap=$ umbrella weighting function; $\rightarrow \cap=$ shifted umbrella; $\operatorname{Max}=$ maximum of the violation areas (Equation 14).

a Simulated data of race model with negative channel correlation. The columns indicate the proportion of $p$ values less than .10 depending on sample size and test procedure. ${ }^{\mathrm{b}}$ Simulated data with a coactivation model (Schwarz, 1994; $\mu_{\mathrm{V}}=0.53, \sigma_{\mathrm{V}}^{2}=18.4, \mu_{\mathrm{A}}=1.34, \sigma_{\mathrm{A}}^{2}=134$, exponential base time with $\mu_{M}=10,20$, and $30 \mathrm{~ms}$ in Blocks 1, 2, and 3, respectively). The columns indicate the proportion of $p$ values less than .05. ${ }^{\mathrm{c}}$ The test is slightly anticonservative for small samples and strata.

effective Type I error under the race model, detection times were generated from antithetic pairs of auditory and visual response times of K.Y. (auditory percentile $P$ was paired with visual percentile $100-P$ ), with the same base time $M$ as for the coactivation model. Table 1 shows that the amount of $p$ values below .10 does not substantially exceed $10 \%$ if the data include at least 30 auditory and visual response times per session.

\section{Discussion}

Detailed investigation of coactivation processes requires variation of the SOA between the stimuli (Miller, 1986; Schwarz, 1994). Even if the researcher is testing only the race model, the SOA at which the largest violation of the race model occurs is not necessarily at $\tau=0$, nor is it known in advance, nor is it the same for all participants. It might thus be necessary to run the experiment with a predetermined set of different SOAs and then integrate the results of the different conditions. Meta-analysis techniques generally require independent $p$ values. This assumption might not be met because the distributions for the redundant conditions at different SOAs are compared with the same unimodal distributions $F_{\mathrm{A}}(t)$ and $F_{\mathrm{V}}(t)$.

In the present note, a straightforward method for integrating the results of race model tests in different experimental conditions (i.e., SOAs) is suggested. Because the entire set of response times is used for the aggregate test statistic, it is more robust than the estimates for single SOA conditions, even if the number of trials per SOA is low. In the nonparametric test (Miller, 1986), extreme results are avoided in which the violation area is 0 or completely outside the range of the simulated areas $(p=.000$; see, e.g., Table
$\mathrm{S} 1$ in the supplementary material). As a potentially useful application, the aggregate test might be used to test the race model in experiments in which the SOA of redundant stimuli is drawn from a continuous distribution. Ulrich and Miller (1997) have shown that the race model predicts that the slope of the SOA function $Z(\tau)=\partial E[T \mid \mathrm{AV}(\tau)] / \partial \tau$ is bounded under the race model: $|Z(\tau)| \leq$ 1 and $Z(\tau)+|Z(-\tau)|<1$. Testing this prediction requires a high number of stimuli with SOA around 0. Using microelectrode recordings, Lakatos, Chen, O'Connell, Mills, \& Schroeder (2007) noticed that tactile stimuli reset the phase of ongoing oscillations in the auditory cortex. Auditory signals arriving at subsequent highexcitability phases are amplified, whereas signals arriving at lowexcitability phases are dampened. Investigating such a relationship in the observed response times requires a variation of the SOA in tiny steps.

Of most importance, the power of the test is substantially increased by assigning specific weights to each SOA before the SOA-specific test statistics are added up. This has been illustrated with different weighting functions (umbrella, shifted umbrella, DSM-based weighting, Table S1). Although, formally, every $\lambda(\tau) \geq 0$ is allowed, appropriate weights substantially increase the power of the significance test. Which $\lambda(\tau)$ should be used? Ideally, the weighting function should be based on a priori considerations (e.g., a plausible and testable coactivation model). As the diffusion superposition model has been shown to successfully predict the response times in Miller (1986), a weighting function based on the DSM seems appropriate here. In speeded response tasks with stimuli of two modalities, the shifted umbrella might serve as a useful nonparametric alternative, because redundancy gains have 
often been shown to be highest if the SOA establishes physiological synchrony of the two stimuli (as opposed to stimulus synchrony; Diederich \& Colonius, 2004; Hershenson, 1962; Miller, 1986; Raab, 1962). In other experimental setups, parametric models might not be available; if plausible arguments for a specific weighting function are lacking, $\lambda(\tau)=1$ might be chosen. Of course, conservative testing requires the weighting function to be specified in advance.

Violation of the race model prediction does not automatically imply that coactivation has taken place. Luce (1986) underlined that the race model inequality is based on both the minimum rule (Equation 1) and context independence. Context independence states that processing of $\mathrm{A}$ in the auditory stimulus is identical to processing of $\mathrm{A}$ in the auditory-visual stimulus. The same holds for the visual subprocess. Therefore, rejection of the race model indicates that either the minimum is incorrect or context independence is not tenable, or both. Most coactivation accounts (e.g., Miller, 1986; Miller \& Ulrich, 2003; Schwarz, 1989, 1994) assume context independence but drop the minimum rule. In contrast, Mordkoff and Yantis (1991) have proposed a model based on the minimum rule-without assuming context independence. The model predicts that processing of a visual target $\mathrm{V}_{i}$ is facilitated if the participant has learned that $\mathrm{V}_{i}$ is more frequent if an auditory stimulus $\mathrm{A}_{j}$ is simultaneously displayed: $P\left(\mathrm{~V}_{i} \mid \mathrm{A}_{j}\right)>P\left(\mathrm{~V}_{i}\right)$. Although the number of stimuli per SOA was constant in Miller (1986), Mordkoff and Yantis (1991, Figure 5) argued that the two sensory channels facilitate each other, because the probability for an auditory signal increases with time since stimulus onset, given that a visual target has appeared. Experiments involving SOA variations as well as manipulations of stimulus contingencies might decide which of these accounts better describes the redundancy gain observed in response times for audiovisual signals.

\section{References}

Bulmer, M. G. (1979). Principles of statistics. New York: Dover.

Davison, A. C., \& Hinkley, D. V. (1997). Bootstrap methods and their application. New York: Cambridge University Press.

Diederich, A., \& Colonius, H. (2004). Bimodal and trimodal multisensory enhancement: Effects of stimulus onset and intensity on reaction time. Perception \& Psychophysics, 66, 1388-1404.

Hershenson, M. (1962). Reaction time as a measure of intersensory facilitation. Journal of Experimental Psychology, 63, 289-293.
Jiang, Y., Rouder, J. N., \& Speckman, P. L. (2004). A note on the sampling properties of the Vincentizing (quantile averaging) procedure. Journal of Mathematical Psychology, 48,186-195.

Kiesel, A., Miller, J., \& Ulrich, R. (2007). Systematic biases and Type I error accumulation in tests of the race model inequality. Behavior Research Methods, 39, 539-551.

Lakatos, P., Chen, C.-M., O’Connell, M. N., Mills, A., Schroeder, C. E. (2007). Neuronal oscillations and multisensory interaction in primary auditory cortex. Neuron, 53, 279-292.

Luce, R. D. (1986). Response times: Their role in inferring elementary mental organization. New York: Oxford University Press.

Maris, G., \& Maris, E. (2003). Testing the race model inequality: A nonparametric approach. Journal of Mathematical Psychology, 47, 507514.

Meredith, M. A., Nemitz, J. W., \& Stein, B. E. (1987). Determinants of multisensory integration in superior colliculus neurons. I. Temporal factors. The Journal of Neuroscience, 7, 3215-3229.

Miller, J. O. (1982). Divided attention: Evidence for coactivation with redundant signals. Cognitive Psychology, 14, 247-279.

Miller, J. O. (1986). Timecourse of coactivation in bimodal divided attention. Perception \& Psychophysics, 40, 331-343.

Miller, J. O., \& Ulrich, R. (2003). Simple reaction time and statistical facilitation: A parallel grains model. Cognitive Psychology, 46, 101151.

Mordkoff, J. T., \& Yantis, S. (1991). An interactive race model of divided attention. Journal of Experimental Psychology: Human Perception and Performance, 17, 520-538.

Raab, D. H. (1962). Statistical facilitation of simple reaction times. Transactions of the New York Academy of Sciences, 24, 574-590.

Saner, H. (1994). A conservative inverse normal test procedure for combining $p$-values in integrative research. Psychometrika, 59, 253-267.

Schwarz, W. (1989). A new model to explain the redundant signals effect. Perception \& Psychophysics, 46, 498-500.

Schwarz, W. (1994). Diffusion, superposition, and the redundant targets effect. Journal of Mathematical Psychology, 38, 504-520.

Townsend, J. T., \& Nozawa, G. (1997). Serial exhaustive models can violate the race model inequality. Implications for architecture and capacity. Psychological Review, 104, 595-602.

Ulrich, R., \& Miller, J. O. (1997). Tests of race models for reaction time in experiments with asynchronous redundant signals. Journal of Mathematical Psychology, 41, 367-381.

Received October 26, 2007

Revision received July 20, 2008

Accepted July 22, 2008 